\title{
Intertwining Multiplication Operators on Function Spaces
}

by

\author{
Bahman YOUSEFI and Leila BAGHERI
}

Presented by Stanistaw KWAPIEN

Summary. Suppose that $X$ is a Banach space of analytic functions on a plane domain $\Omega$. We characterize the operators $T$ that intertwine with the multiplication operators acting on $X$.

Introduction. Suppose that the set of analytic polynomials is dense in a Banach space $X$ of functions analytic on a plane domain $\Omega$, and suppose that for each $\lambda \in \Omega$ the linear functional of evaluation at $\lambda, e(\lambda)$, is bounded on $X$. We further assume that $X$ contains the constant functions and that multiplication by the independent variable $z$ defines a bounded linear operator $M_{z}$ on $X$. Also, we assume that for any fixed $n \in \mathbb{N}$, every $f$ in $X$ has a unique decomposition $f=\bigoplus_{i=0}^{n-1} f_{i}$ where $f_{i} \in X_{i}$ and $X_{i}$ is the closed linear span of the set $\left\{z^{n k+i}: k \geq 0\right\}$ in $X$ for $i=0,1, \ldots, n-1$.

The weighted Hardy spaces, $H^{p}(\beta)$, are examples of such spaces. For more information on such spaces see $[5,7,8]$.

Throughout this paper, by a Banach space of analytic functions on a plane domain $\Omega$ we mean one satisfying the above conditions. For some results on such spaces see $[1,2,3,6,10]$.

A complex-valued function $\varphi$ on $\Omega$ for which $\varphi f \in X$ for every $f \in X$ is called a multiplier of $X$. Every multiplier $\varphi$ of $X$ determines a multiplication operator $M_{\varphi}$ on $X$ by $M_{\varphi} f=\varphi f, f \in X$. The set of all multipliers of $X$ is denoted by $M(X)$. Clearly $M(X) \subset H^{\infty}(\Omega)$, where $H^{\infty}(\Omega)$ is the space of all bounded analytic functions on $\Omega$. In fact $\|\varphi\|_{\infty} \leq\left\|M_{\varphi}\right\|$. A good source on this topic is [4].

2000 Mathematics Subject Classification: Primary 47B35; Secondary 47B38.

Key words and phrases: Banach spaces of analytic functions, bounded point evaluation, Fredholm alternative, multiplication operators. 
Let $B(X)$ be the set of all bounded operators on $X$. If $T \in B(X)$ and $T M_{\varphi}=-M_{\varphi} T$ or $T M_{\varphi^{2}}=M_{\varphi^{2}} T$ where $\varphi \in H^{\infty}(\Omega)$, then under suitable conditions the structure of $T$ was determined in [9]. In this paper we want to characterize the operators $T$ satisfying $T M_{\varphi}=M_{\psi} T$ where $\varphi$ and $\psi$ are multipliers of $X$. Specially, the case $\psi=a \varphi$ is considered.

Main results. In this section we will characterize the structure of operators that intertwine with the multiplication operators acting on function spaces.

Note that by our assumptions each $f \in X$ has a unique decomposition $f=\bigoplus_{i=0}^{n-1} f_{i}$ where $f_{i} \in X_{i}$ and $X_{i}$ is the closed linear span of the set $\left\{z^{n k+i}: k \geq 0\right\}$ in $X$ for $i=0, \ldots, n-1$. From now on suppose that $X$ is a Banach space of analytic functions on the open unit disc $U$ and $0<|a| \leq 1$. Assume further that the composition operators $C_{\varphi}$ and $C_{a \varphi}$ are bounded on $X$ where $\varphi$ is a multiplier of $X$.

TheOREM 1. Suppose that $\varphi$ is a multiplier of $X$ and let $T \in B(X)$ be such that $T M_{\varphi^{n}}=a^{n} M_{\varphi^{n}} T$ for some positive integer $n$. Also consider $f$ in $X$ with decomposition $\bigoplus_{i=0}^{n-1} f_{i}$ where $f_{i} \in X_{i}$ for $i=0, \ldots, n-1$. Then there exist functions $u_{0}, u_{1}, \ldots, u_{n-1}$ such that

$$
T C_{\varphi} f=u_{0} C_{a \varphi} f_{0}+u_{1} C_{a \varphi} f_{1}+\cdots+u_{n-1} C_{a \varphi} f_{n-1} .
$$

Proof. Let $u_{0}=T(1)$ and put

$$
\psi_{i}=\left(T M_{\varphi^{i}}-a^{i} M_{\varphi^{i}} T\right)(1)
$$

for $i=1, \ldots, n-1$. For all integers $k \geq 0$ we have

$$
\begin{aligned}
T C_{\varphi} z^{n k} & =T\left(\varphi^{n k}\right)=\left(T M_{\varphi}^{n k}\right)(1) \\
& =\left(a^{n k} M_{\varphi}^{n k} T\right)(1)=u_{0} a^{n k} C_{\varphi} z^{n k}=u_{0} C_{a \varphi} z^{n k} .
\end{aligned}
$$

Also, for all $i=1, \ldots, n-1$, by using $(*)$ we get

Therefore

$$
\begin{aligned}
T C_{\varphi} z^{i}=T\left(\varphi^{i}\right)=\left(T M_{\varphi^{i}}\right)(1) & =\psi_{i}+\left(a^{i} M_{\varphi^{i}} T\right)(1) \\
& =\psi_{i}+u_{0} a^{i} C_{\varphi} z^{i} \\
& =\psi_{i}+u_{0} C_{a \varphi} z^{i} .
\end{aligned}
$$

$$
\begin{aligned}
T C_{\varphi} z^{n k+i} & =\left(T M_{\varphi}^{n k+i}\right)(1) \\
& =a^{n k} M_{\varphi}^{n k}\left(\psi_{i}+u_{0} C_{\varphi} C_{a z} z^{i}\right) \\
& =\psi_{i} C_{\varphi} C_{a z} z^{n k}+u_{0} C_{\varphi} C_{a z} z^{n k+i} \\
& =\left(u_{0}+\frac{\psi_{i}}{(a \varphi)^{i}}\right) C_{a \varphi} z^{n k+i}
\end{aligned}
$$


for all integers $k \geq 0$ and $i=1, \ldots, n-1$. Now consider a polynomial $p$ with decomposition $p=\bigoplus_{i=0}^{n-1} p_{i}$ where $p_{i} \in X_{i}$ for $i=0, \ldots, n-1$. So we have

$$
\begin{aligned}
T C_{\varphi} p & =T C_{\varphi} p_{0}+T C_{\varphi} p_{1}+\cdots+T C_{\varphi} p_{n-1} \\
& =u_{0} C_{a \varphi} p_{0}+u_{1} C_{a \varphi} p_{1}+\cdots+u_{n-1} C_{a \varphi} p_{n-1}
\end{aligned}
$$

where $u_{i}=u_{0}+\psi_{i} /(a \varphi)^{i}$ for $i=1, \ldots, n-1$. Since the set of analytic polynomials is dense in $X$, we get

$$
T C_{\varphi} f=u_{0} C_{a \varphi} f_{0}+u_{1} C_{a \varphi} f_{1}+\cdots+u_{n-1} C_{a \varphi} f_{n-1} .
$$

This completes the proof.

The following corollary is an immediate consequence of the proof of Theorem 1, which gives the structure of the functions $u_{i}$ in Theorem 1 .

Corollary 2. Under the conditions of Theorem 1 , for $f=\bigoplus_{i=0}^{n-1} f_{i}$ in $X$, we have

$$
T C_{\varphi} f=T(1) C_{a \varphi} f_{0}+\sum_{i=1}^{n-1}\left(T(1)+\frac{\left(T M_{\varphi^{i}}-a^{i} M_{\varphi^{i}} T\right)(1)}{(a \varphi)^{i}}\right) C_{a \varphi} f_{i} .
$$

THEOREM 3. Let $\varphi$ be a multiplier of $X$ and $T \in B(X)$ be such that $T M_{\varphi^{n}}=a^{n} M_{\varphi^{n}} T$. If $C_{a \varphi}$ is invertible and $T M_{\varphi}-a M_{\varphi} T$ is compact, then $T C_{\varphi}=M_{u_{0}} C_{a \varphi}$ where $u_{0}=T(1)$.

Proof. Let $f \in X$ and $f=\bigoplus_{i=0}^{n-1} f_{i}$ where $f_{i} \in X_{i}$ for $i=0,1, \ldots, n-1$. By Theorem 1, we have

$$
T C_{\varphi} f=u_{0} C_{a \varphi} f_{0}+u_{1} C_{a \varphi} f_{1}+\cdots+u_{n-1} C_{a \varphi} f_{n-1}
$$

where

$$
\begin{aligned}
u_{0} & =T(1) \\
u_{i} & =u_{0}+\psi_{i} /(a \varphi)^{i} \\
\psi_{i} & =\left(T M_{\varphi^{i}}-a^{i} M_{\varphi^{i}} T\right)(1)
\end{aligned}
$$

for $i=1, \ldots, n-1$. Put

$$
S=\left(T M_{\varphi}-a M_{\varphi} T\right) C_{\varphi}
$$

Then $S$ is compact and we have

$$
\begin{aligned}
S f= & T C_{\varphi}(z f)-a M_{\varphi} T C_{\varphi} f \\
= & u_{0} C_{a \varphi}\left(z f_{n-1}\right)+u_{1} C_{a \varphi}\left(z f_{0}\right)+\cdots+u_{n-1} C_{a \varphi}\left(z f_{n-2}\right) \\
& -a \varphi\left(u_{0} C_{a \varphi} f_{0}+\cdots+u_{n-1} C_{a \varphi} f_{n-1}\right) .
\end{aligned}
$$

Now by substituting $u_{0}+\psi_{i} /(a \varphi)^{i}$ for $u_{i}$ in the above relation, we get

$$
\begin{aligned}
S f= & { }_{1} C_{a \varphi} f_{0}+\left(\frac{\psi_{2}}{a \varphi}-\psi_{1}\right) C_{a \varphi} f_{1}+\cdots \\
& +\left(\frac{\psi_{n-1}}{(a \varphi)^{n-2}}-\frac{\psi_{n-2}}{(a \varphi)^{n-3}}\right) C_{a \varphi} f_{n-2}-\frac{\psi_{n-1}}{(a \varphi)^{n-2}} C_{a \varphi} f_{n-1}
\end{aligned}
$$


Since $S$ is compact, so also are

$$
\left.M_{\psi_{1}} C_{a \varphi}\right|_{X_{0}}=\left.S\right|_{X_{0}} \quad \text { and }\left.\quad M_{\psi_{1}} M_{(a \varphi)^{n}} C_{a \varphi}\right|_{X_{0}} .
$$

But

$$
\left.M_{\psi_{1}} M_{(a \varphi)^{n}} C_{a \varphi}\right|_{X_{i}}=\left.\left.M_{(a \varphi)^{i}} M_{\psi_{1}} C_{a \varphi}\right|_{X_{0}} M_{z^{n-i}}\right|_{X_{i}},
$$

and thus indeed $M_{\psi_{1}(a \varphi)^{n}} C_{a \varphi}$ is compact on $X$. This implies that $M_{\psi_{1}(a \varphi)^{n}}$ is compact on $X$, since $C_{a \varphi}$ is invertible. Now by the Fredholm alternative we get $(a \varphi)^{n} \psi_{1}=0$, which implies that $\psi_{1}=0$, because $a \varphi$ is univalent. By the same method we can see that $\psi_{1}=\psi_{2}=\cdots=\psi_{n-1}=0$, so $u_{i}=u_{0}$ for all $i=1, \ldots, n-1$. Now $(* *)$ implies that $T C_{\varphi}=M_{u_{0}} C_{a \varphi}$ and this completes the proof.

Corollary 4. Let $T \in B(X)$ be such that $T M_{z^{n}}=a^{n} M_{z^{n}} T$ where $0<|a| \leq 1$. If $T M_{z}-a M_{z} T$ is compact, then $T=M_{u_{0}} C_{a z}$ where $u_{0}=T(1)$.

\section{References}

[1] P. S. Bourdon and J. H. Shapiro, Spectral synthesis and common cyclic vectors, Michigan Math. J. 37 (1990), 71-90.

[2] B. Khani Robati, On the structure of certain operators on spaces of analytic functions, Asian J. Math., submitted.

[3] S. Richter, Invariant subspaces in Banach spaces of analytic functions, Trans. Amer. Math. Soc. 304 (1987), 585-616.

[4] A. Shields and L. Wallen, The commutants of certain Hilbert space operators, Indiana Univ. Math. J. 20 (1971), 777-788.

[5] B. Yousefi, Bounded analytic structure of the Banach spaces of formal power series, Rend. Circ. Mat. Palermo 51 (2002), 403-410.

[6] -, Multiplication operators on Hilbert spaces of analytic functions, Arch. Math. (Basel) 83 (2004), 536-539.

[7] -, Strictly cyclic algebra of operators acting on Banach spaces $H^{p}(\beta)$, Czechoslovak Math. J. 54 (129) (2004), 261-266.

[8] -, On the eighteenth question of Allen Shields, Internat. J. Math. 16 (2005), 37-42.

[9] B. Yousefi and S. Foroutan, On the multiplication operators on spaces of analytic functions, Studia Math. 168 (2005), 187-191.

[10] K. Zhu, Irreducible multiplication operators on spaces of analytic functions, J. Operator Theory 51 (2004), 377-385.

Bahman Yousefi

Department of Mathematics

College of Sciences

Shiraz University

Shiraz 71454, Iran

E-mail: byousefi@shirazu.ac.ir
Leila Bagheri Department of Mathematics Shiraz Payame-Noor University

Shiraz, Iran E-mail: sky_sun_1980@yahoo.com 